\title{
Autonomous platform for life-critical decision support in the ICU
}

\author{
Kristof Steurbaut and Filip De Turck \\ Department of Information Technology (INTEC), Ghent University - IBBT, \\ Gaston Crommenlaan 8 Bus 201, 9050 Gent, Belgium \\ Kristof.Steurbaut@intec.UGent.be
}

\begin{abstract}
The Intensive Care Unit is a complex, data-intensive and critical environment in which the adoption of Information Technology is growing. As physicians become more dependent on the computing technology to support decisions, raise real-time alerts and notifications of patient-specific conditions, this software has strong dependability requirements. The dependability challenges are expressed in terms of availability, reliability, performance, usability and maintenance of the system. Our research focuses on the design and development of a generic autonomous ICU service platform. COSARA is a computer-based platform for infection surveillance and antibiotic management in ICU. During its design, development and evaluation, we identified both technological and human factors that affect robustness. We presented the identified research questions that will be addressed in detail during $\mathrm{PhD}$ research.
\end{abstract}

Keywords: Dependability, services, ICU

\section{Introduction}

The computerization of medical decision support processes and clinical guidelines contributes to better quality of care [1]. Decisions can be based on large amounts of patient-specific data and computing power is used to execute complex clinical guidelines. The aim of our Intensive Care Unit (ICU) service platform is to provide Computer-based Surveillance and Alerting of Nosocomial Infections, Antimicrobial Resistance and Antibiotic Consumption (COSARA) [2]. The COSARA platform collects and combines data from existing clinical sources such as laboratory database, Picture Archiving and Communication System (PACS), Intensive Care Information System (ICIS) and antibiotics registrations [3]. COSARA offers an overview of all antibiotic prescriptions, infection registrations, microbiology and related clinical parameters in one single application. Even information which was not present in the originating systems is registered, for example by requesting physicians' motivation for starting an antibiotic therapy. Advanced decision support services can be created on the platform, to notify about antibiotic dosage or organ failure. As the application is entirely integrated in the physicians' daily workflow, physicians become more dependent on this patient-specific visualisation and decision support functionality. 


\section{Motivation}

The design and development of a dependable ICU service platform is investigated. Dependability of the software system is the ability to deliver service that can justifiably be trusted. It encompasses a combination of reliability, availability, confidentiality, integrity, maintainability, safety. The alternate definition is to avoid service failures that are more frequent and more severe than acceptable [5]. With the introduction of advanced clinical decision support services, healthcare is becoming more dependent on software. Most of the quality attributes were not directly measurable, but were expressed as perceived by the end users. Our aim is to provide a platform which can autonomously react on unexpected failures, while ensuring the high level of dependability. As physicians do not have the technical expertise to reconfigure or redeploy the system, the system should autonomously detect failures and self-configure or self-manage the services, even without the intervention of the IT developer. In Figure 1 we identified the impact of issues from the COSARA evaluation: (i) data inaccuracy, (ii) integration mismatches, (iii) human registration inaccuracy, (iv) slow system response, (v) link failures, (vi) software server crash, (vii) hardware and power failures. Data inaccuracy is the result of unavailable data, missing data or invalid data. Integration mismatches occur when wrong or different patient identifications are used in the system.

\section{Automomous Service Platform}

Unlike traditional approaches, such as logging system failures and keeping an audit trail of all interactions, the system should anticipate on unexpected failures by reconfiguring, recovering the services. In order to obtain a fully autonomous computer-based ICU service platform, we formulated our research questions:

- How can the COSARA software platform support dependability in the best possible way?
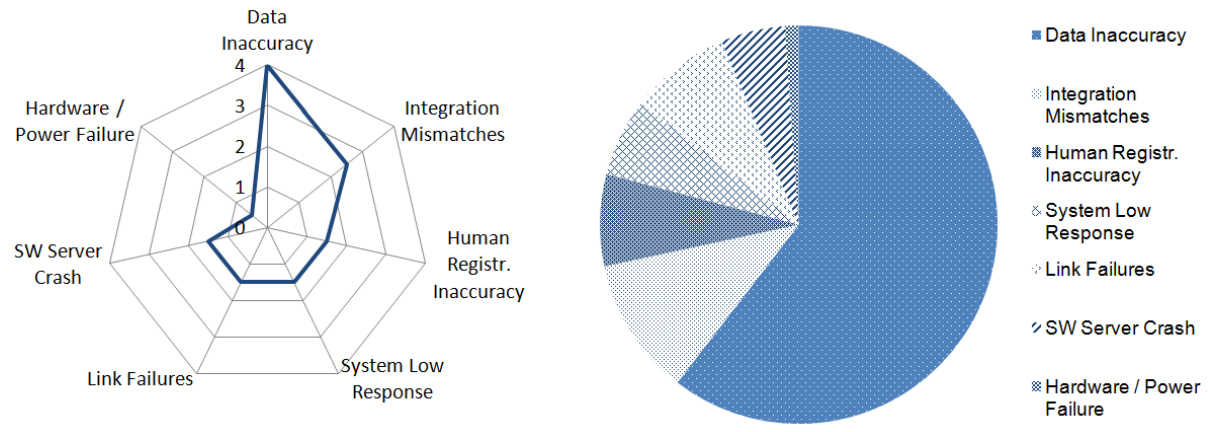

Fig. 1. Evaluation of COSARA reliability: importance of the observed issues (left) and the relative number of issue reports (right) 


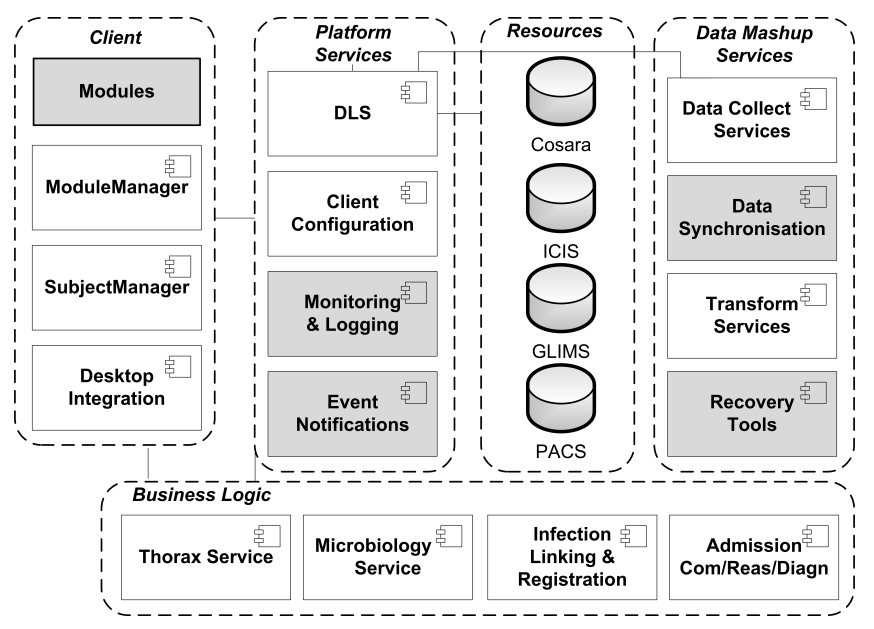

Fig. 2. COSARA Platform Architecture

- What platform components are necessary in the platform design?

The architecture consists of Data Mashup Services, Platform Services, Business Logic Services and Client Modules. Fig. 2 highlights the necessary components to achieve an autonomous service platform.

- How to monitor the system health and service performance?

A monitoring component allows to track the system health and status of all running bedside clients. Database performance is measurable because the execution time of each query is logged. The client can be restarted if it appears in off-line status. From the physicians' perspective, time delays, for example to display large images, were associated with a complete malfunctioning of the application. The user was unaware of the delay and expected a failure of the system. Usability and notications about the system behavior are crucial.

- How to realize autonomous synchronisation with external resources?

The physician and developer are notified of detected fault states by tracking service variables. The availability of the communication links between the data sources (such as lab or PACS) must be high. When a link breaks, the ICU administrator is notified about this failure. In case of failures, a flexible recovery mechanism can restart all services.

- How to ensure high level of data quality?

In the resources (ICIS, GLIMS-Lab, PACS), data might not be available in a well-structured format, might be missing or become available at a later moment. The data quality is of major importance and has to be up-to-date and display the same information as the existing systems. Human factors can influence the data accuracy. For example, registering different patientidentifications across systems, can cause mismatches of data. Registrations should be correctly entered in the system. Inaccurate data could result in wrong decisions and offering only valid data creates trust among its users. 
Therefore a data synchronisation mechanism is needed between the existing clinical data sources and the COSARA database. The data collection process and synchronisation use the Data Lookup Service (DLS) to query the databases. This DLS is monitored and in case of low performance, the load has to be spread across different DLSs.

- How to adapt the platform to changes in business logic and data persistence? Some assumptions about the presence of clinical values and identifications were made in the initial design. However, during operation, it was noticed that some identifications could be missing and were added after the patient was admitted a couple of days.

\section{Validation}

The COSARA application, which has been deployed on 56 bedside PCs in the Intensive Care Unit of Ghent University Hospital since April 2010, was evaluated during a 5-month period. The prototype was evaluated by a study nurse and the data quality was validated by all ICU researchers. We are now planning to further apply dependability mechanisms to the platform.

Acknowledgments. This research has been done within the context of the COSARA project, which was supported by the Institute for Science and Technology in Flanders (IWT).

\section{References}

1. Damiani, G., Pinnarelli, L., Colosimo, S., Almiento, R., Sicuro, L., Galasso, R., Sommella, L., Ricciardi, W.: The effectiveness of computerized clinical guidelines in the process of care: a systematic review. BMC Health Services Research 10:2 (2010)

2. Steurbaut, K., Van Hoecke, S., Colpaert, K., Lamont, K., Taveirne, K., Depuydt, P., Benoit, D., Decruyenaere, J., De Turck, F.: Use of web services for computerized medical decision support, including infection control and antibiotic management, in the intensive care unit. . J. Telemed Telecare 16, 25-29 (2010)

3. Steurbaut, K., Van Hoecke, S., Taveirne, K., Lamont, K., De Turck, F., Colpaert, K., Depuydt, P., Benoit, D., Danneels C., Decruyenaere, J.: Design of Software Services for Computer-Based Infection Control and Antibiotic Management in the Intensive Care Unit. In: International Conference on eHealth, Telemedicine, and Social Medicine (2009)

4. Avizienis, A., Laprie, J., Randell, B., Landwehr, C.: Basic Concepts and Taxonomy of Dependable and Secure Computing. IEEE Transactions on dependable and secure computing Vol. 1 No. 1 (2004) 